\title{
AN ANALYTICAL AND NUMERICAL STUDY OF UNSTEADY CHANNEL FLOW WITH SLIP
}

\author{
MICCAL T. MATTHEWS ${ }^{\otimes 1}$ and KAREN M. HASTIE ${ }^{1}$
}

(Received 28 April, 2012; revised 18 October, 2012; first published online 8 February, 2013)

\begin{abstract}
A theoretical investigation of the unsteady flow of a Newtonian fluid through a channel is presented using an alternative boundary condition to the standard no-slip condition, namely the Navier boundary condition, independently proposed over a hundred years ago by both Navier and Maxwell. This boundary condition contains an extra parameter called the slip length, and the most general case of a constant but different slip length on each channel wall is studied. An analytical solution for the velocity distribution through the channel is obtained via a Fourier series, and is used as a benchmark for numerical simulations performed utilizing a finite element analysis modified with a penalty method to implement the slip boundary condition. Comparison between the analytical and numerical solution shows excellent agreement for all combinations of slip lengths considered.
\end{abstract}

2010 Mathematics subject classification: 76D05.

Keywords and phrases: channel flow, slip, Navier boundary condition, finite element, penalty method.

\section{Introduction}

A major theme during the last several decades has been miniaturization down to the micro and nano scales. The study of small-scale fluid flow is of fundamental importance due to the necessity of understanding the nature of flows at this scale [5]. The first question to be addressed is: what happens when the thickness of a liquid film becomes comparable to the size of the molecules themselves? It has been demonstrated that physical and mechanical properties cannot be understood by extrapolating those of the bulk fluid $[2,7]$. One of the main reasons for this is that when devices are scaled down, the surface-to-volume ratio increases and therefore surface-related phenomena become increasingly important. Qualitatively new features

\footnotetext{
${ }^{1}$ School of Engineering, Edith Cowan University, Joondalup, WA 6027, Australia; e-mail: miccal.matthews@ecu.edu.au, khastie0@gmail.com.

(C) Australian Mathematical Society 2013, Serial-fee code 1446-1811/2013\$16.00
} 
emerge when physical systems are downsized, and it becomes important to understand the various types of interactions in the flow and their underlying physical mechanisms, such as the interaction between the constituents of the flow and solid boundaries.

An example of the breakdown of conventional macroscopic ideas at small scales is the no-slip boundary condition between a fluid and a solid [1, Ch. 3]. The boundary condition illustrating how momentum is transferred from the fluid to the solid can vary from stick to slip in response to the physical/chemical properties of the solid surface [10, Ch. 2]. In particular, the traditional Navier-Stokes model of fluid flow with a no-slip boundary condition at a fluid-solid interface requires the following [6].

(i) The fluid is a continuum, which is always satisfied since there are more than 1 million molecules in the smallest volume in which appreciable macroscopic changes take place.

(ii) The flow is near thermodynamic equilibrium, which is satisfied if there are a sufficient number of molecular encounters during a time period that is small compared to the smallest time scale at which the flow changes. During this time period, a molecule would have moved a distance that is small compared to the smallest length scale of the flow.

The traditional no-slip condition at a fluid-solid interface, namely that the fluid velocity is zero relative to the solid boundary, requires condition (ii) to be satisfied. For the fluid flow to be in thermal equilibrium with an adjacent solid surface, an infinitely high frequency of fluid particle collisions with the solid boundary is required. In a small-scale system, the collision frequency is not high enough to ensure thermodynamic equilibrium, resulting in a certain degree of tangential velocity slip. A simple modification to allow a certain degree of tangential velocity slip is to replace the no-slip boundary condition [5, 6]. Navier [15] introduced the linear boundary condition, later proposed independently by Maxwell [14], which is a standard characterization of slip; the component of the fluid velocity tangent to the surface is proportional to the rate of strain and the constant of proportionality is called the slip length.

In this paper, we model the unsteady/startup flow between two parallel plates with differing amounts of slip on each plate via an analytical solution, which is then used as a benchmark for numerical simulations using a finite element method that employs the slip boundary conditions via a penalty implementation. Although the problem might seem academic in nature, channel flows are fairly common in micro- and nanoelectromechanical systems and lab-on-a-chip technologies. There have been numerous analytical studies of unsteady Navier-Stokes flow with the standard no-slip boundary condition (a review of these exact solutions is given by Wang [18]), but the application of Navier slip conditions to an unsteady flow is only valid for parallel flows, such as the unsteady flow through a tube [12] and the problem considered here. For studies with flow reversal such as unsteady oscillatory flow, Wang [19] has shown that the Navier slip condition is not valid. 


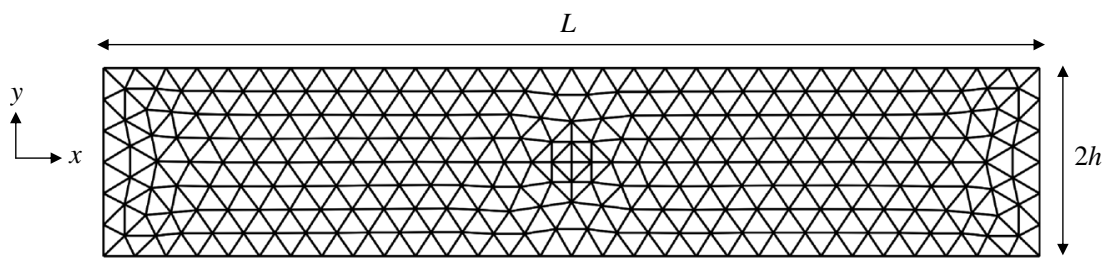

FIgure 1. Schematic diagram of the channel with length $L$ and width $2 h$. The coordinate system used for the analytical and computational solution is shown. An example of the mesh used for the numerical solution is also shown, corresponding to 496 elements.

The analytical solution characterizing the flow is derived via Fourier series in Section 2. The numerical method is described and verified via steady no-slip and slip solutions from the literature in Section 3. A discussion of the unsteady no-slip and slip results is given in Section 4. Appendix A deals with certain mathematical details of the analytical solution.

\section{Analytical solution}

Consider the unsteady flow of an incompressible Newtonian fluid between two parallel planes of infinite extent separated by a distance $2 h$ as illustrated in Figure 1. Initially the fluid is at rest, and is set in motion by a suddenly imposed and maintained pressure difference $P_{0}-P_{L}$ acting over a distance $L$ parallel to the planes. A Cartesian coordinate system $\left(x^{*}, y^{*}, z^{*}\right)$ is used, where $y^{*}$ is measured from the midpoint between the two planes, normal to the planes, while $x^{*}$ is measured parallel to the planes and is positive in the direction of flow. A velocity distribution of the form

$$
v_{x}^{*}=v_{x}^{*}\left(y^{*}, t^{*}\right), \quad v_{y}^{*}=0, \quad v_{z}^{*}=0
$$

is assumed so that mass conservation is automatically satisfied and the $x^{*}$ component of momentum conservation may be written as

$$
\rho \frac{\partial v_{x}^{*}}{\partial t^{*}}=-\frac{\partial P^{*}}{\partial x^{*}}+\mu \frac{\partial^{2} v_{x}^{*}}{\partial y^{* 2}}
$$

while the $y^{*}$ and $z^{*}$ components yield $\partial P^{*} / \partial y^{*}=0=\partial P^{*} / \partial z^{*}$. Thus the $x^{*}$ component may be written as

$$
\frac{d P^{*}}{d x^{*}}=-\rho \frac{\partial v_{x}^{*}}{\partial t^{*}}+\mu \frac{\partial^{2} v_{x}^{*}}{\partial y^{* 2}} .
$$

Since the left-hand side of the above equation is a function of $x^{*}$ only and the righthand side is a function of $y^{*}$ and $t^{*}$ only,

$$
\frac{d P^{*}}{d x^{*}}=-A=-\rho \frac{\partial v_{x}^{*}}{\partial t^{*}}+\mu \frac{\partial^{2} v_{x}^{*}}{\partial y^{* 2}},
$$


where $A$ is a constant. By applying the boundary conditions $P^{*}=P_{0}$ at $x^{*}=0$ and $P^{*}=P_{L}$ at $x^{*}=L$, we find that

$$
P^{*}=-A x^{*}+P_{0}, \quad A=\frac{P_{0}-P_{L}}{L}>0 .
$$

Hence

$$
\rho \frac{\partial v_{x}^{*}}{\partial t^{*}}=A+\mu \frac{\partial^{2} v_{x}^{*}}{\partial y^{* 2}},
$$

which must be solved subject to the following initial condition and slip boundary conditions given by the Navier boundary condition:

$$
\begin{aligned}
& t^{*}=0: v_{x}^{*}=0 \quad \text { for }-h \leq y^{*} \leq h, \\
& y^{*}=\mp h: v_{x}^{*} \mp \ell^{* \mp} \frac{\partial v_{x}^{*}}{\partial y^{*}}=0 \quad \text { for } t^{*} \geq 0,
\end{aligned}
$$

where $\ell^{* \mp}>0$ are the slip lengths on the bottom and top plates, respectively. Taking $\ell^{* \mp}=0$ yields the no-slip condition, while the case of infinite slip lengths corresponds to a potential flow situation.

By introducing the dimensionless variables

$$
v_{x}=\frac{2 \mu v_{x}^{*}}{A h^{2}}, \quad y=\frac{y^{*}}{h}, \quad t=\frac{\mu t^{*}}{\rho h^{2}}, \quad \ell^{\mp}=\frac{\ell^{* \mp}}{h},
$$

the problem to be solved becomes the partial differential equation (PDE)

$$
\frac{\partial v_{x}}{\partial t}=2+\frac{\partial^{2} v_{x}}{\partial y^{2}}
$$

subject to the initial condition

$$
t=0: v_{x}=0 \text { for }-1 \leq y \leq 1
$$

and boundary conditions

$$
y=\mp 1: v_{x} \mp \ell^{\mp} \frac{\partial v_{x}}{\partial y}=0 \text { for } t \geq 0 .
$$

The PDE (2.3) is not in a form that can be solved via separation of variables, but by assuming that the solution is of the form $v_{x}(y, t)=v_{x, \infty}(y)-u(y, t)$, where $v_{x, \infty}(y)$ is the steady-state velocity profile and $u(y, t)$ is the transient velocity distribution, the governing PDE may be transformed into an ordinary differential equation (ODE) for the steady solution and a PDE for the transient solution that is amenable to separation of variables. In particular, we have the ODE system

$$
\begin{aligned}
& \frac{d^{2} v_{x, \infty}}{d y^{2}}=-2, \\
& y=\mp 1: v_{x, \infty} \mp \ell^{\mp} \frac{d v_{x, \infty}}{d y}=0,
\end{aligned}
$$


and the PDE system

$$
\begin{aligned}
& \frac{\partial u}{\partial t}=\frac{\partial^{2} u}{\partial y^{2}}, \\
& t=0: u=v_{x, \infty}(y), \\
& y=\mp 1: u \mp \ell^{\mp} \frac{\partial u}{\partial y}=0 .
\end{aligned}
$$

2.1. Steady-state solution The solution to the ODE system (2.4), (2.5) is found to be

$$
v_{x, \infty}(y)=\frac{3\left(\ell^{-}+\ell^{+}\right)+4 \ell^{-} \ell^{+}+2}{\ell^{-}+\ell^{+}+2}-\frac{2\left(\ell^{-}-\ell^{+}\right)}{\ell^{-}+\ell^{+}+2} y-y^{2} .
$$

Notice that when $\ell^{-}=\ell^{+}=0$ (that is, no slip on the channel walls), equation (2.6) reduces to the standard parabolic profile $v_{x, \infty}(y)=1-y^{2}$, and when $\ell^{-}=\ell^{+}=\ell$ (equal slip on the channel walls) it reduces to $v_{x, \infty}(y)=1+2 \ell-y^{2}$ as in the paper by Matthews and Hill [11].

2.2. Transient solution With the steady solution given by equation (2.6), the PDE system becomes

$$
\begin{aligned}
& \frac{\partial u}{\partial t}=\frac{\partial^{2} u}{\partial y^{2}}, \\
& u(y, 0)=\frac{3\left(\ell^{-}+\ell^{+}\right)+4 \ell^{-} \ell^{+}+2}{\ell^{-}+\ell^{+}+2}-\frac{2\left(\ell^{-}-\ell^{+}\right)}{\ell^{-}+\ell^{+}+2} y-y^{2}, \\
& u(\mp 1, t) \mp \ell^{\mp} \frac{\partial u}{\partial y}(\mp 1, t)=0 .
\end{aligned}
$$

By applying the change of variable $\hat{y}=y+1$, we obtain

$$
\begin{aligned}
& \frac{\partial u}{\partial t}=\frac{\partial^{2} u}{\partial \hat{y}^{2}}, \\
& u(\hat{y}, 0)=\frac{4 \ell^{-}\left(1+\ell^{+}\right)}{\ell^{-}+\ell^{+}+2}+\frac{4\left(1+\ell^{+}\right)}{\ell^{-}+\ell^{+}+2} \hat{y}-\hat{y}^{2}, \\
& u(0, t)-\ell^{-} \frac{\partial u}{\partial \hat{y}}(0, t)=0, \quad u(2, t)+\ell^{+} \frac{\partial u}{\partial \hat{y}}(2, t)=0 .
\end{aligned}
$$

This system bears resemblance to the problem of heat conduction in a finite medium (of width "2") at a prescribed initial temperature with Newtonian heat loss at each boundary [3, Ch. 3]. The solution to this problem is detailed by Hill and Dewynne [8, Ch. 4] with equal thermal coefficients in the Newtonian heat loss boundary conditions, and as such only the main solution steps are illustrated here.

By assuming a solution of the form $u(\hat{y}, t)=Y(\hat{y}) T(t)$ we obtain the ODEs

$$
Y^{\prime \prime}(\hat{y})+\lambda Y(\hat{y})=0, \quad T^{\prime}(t)+\lambda T(t)=0,
$$


where $\lambda$ is a separation constant. The solution for $T(t)$ is simply $T(t)=A e^{-\lambda t}$, where $A$ is an integration constant. To ensure that the solution is bounded as $t \rightarrow \infty$, we require $\lambda=k^{2}>0$; that is, the separation constant must be positive (it may also be shown that the separation constant must be positive for the boundary value problem for $Y(\hat{y})$ to have a unique nontrivial solution: see Appendix A). Then the ODE and boundary conditions for $Y(\hat{y})$ become the Sturm-Liouville boundary value problem

$$
\begin{aligned}
& Y^{\prime \prime}(\hat{y})+k^{2} Y(\hat{y})=0, \\
& Y(0)-\ell^{-} Y^{\prime}(0)=0, \quad Y(2)+\ell^{+} Y^{\prime}(2)=0 .
\end{aligned}
$$

The solution for $Y(\hat{y})$ is found to be

$$
Y(\hat{y})=\sin (k \hat{y})+\ell^{-} k \cos (k \hat{y}),
$$

where $k$ is a root of

$$
f(k)=\tan (2 k)-\frac{k\left(\ell^{-}+\ell^{+}\right)}{\ell^{-} \ell^{+} k^{2}-1}=0 .
$$

The transcendental equation (2.7) has infinitely many solutions which may be found by any standard root-finding algorithm, and without loss of generality we may consider just the positive solutions since $f(k)$ is an odd function of $k$.

By the principle of superposition,

$$
u(\hat{y}, t)=\sum_{n=1}^{\infty} Y_{n}(\hat{y}) T(t)=\sum_{n=1}^{\infty} A_{n}\left[\sin \left(k_{n} \hat{y}\right)+\ell^{-} k_{n} \cos \left(k_{n} \hat{y}\right)\right] e^{-k_{n}^{2} t},
$$

where $0<k_{1}<k_{2}<\cdots$ are the eigenvalues from equation (2.7) arranged in ascending order. By applying the initial condition and using the orthogonality condition

$$
\int_{0}^{2} Y_{m}(\hat{y}) Y_{n}(\hat{y}) d \hat{y}=0 \quad \text { for } m \neq n
$$

as well as equation (2.7), after a considerable amount of algebraic manipulation we find that the constants $A_{n}$ may be determined from

$$
\begin{gathered}
A_{n}\left\{\frac{2\left(\ell^{-}\right)^{2}\left(\ell^{+}\right)^{2} k_{n}^{4}+\left[\left(\ell^{-}\right)^{2}\left(\ell^{+}+2\right)+\left(\ell^{+}\right)^{2}\left(\ell^{-}+2\right)\right] k_{n}^{2}+\ell^{-}+\ell^{+}+2}{2\left[k_{n}^{2}\left(\ell^{+}\right)^{2}+1\right]}\right\} \\
=\frac{2}{k_{n}^{3}}+\sin \left(2 k_{n}\right)\left[\frac{4 \ell^{-} \ell^{+}\left(\ell^{-}+1\right)}{\ell^{-}+\ell^{+}+2}+\frac{2 \ell^{-}\left(\ell^{-}+\ell^{+}\right)-4}{k_{n}^{2}\left(\ell^{-}+\ell^{+}+2\right)}\right] \\
\quad-\frac{\cos \left(2 k_{n}\right)}{k_{n}}\left[\frac{4 \ell^{-}\left(\ell^{-}+\ell^{+}+1\right)+4 \ell^{+}}{\ell^{-}+\ell^{+}+2}+\frac{2}{k_{n}^{2}}\right] .
\end{gathered}
$$

Notice that $Y_{n}$ and the expression for $A_{n}$ are odd functions of $k_{n}$, so that there is indeed no loss of generality in only using the positive eigenvalues from (2.7). 
TABLE 1 . The first five eigenvalues $k_{n}$ and Fourier coefficients $A_{n}$ for various amounts of slip.

\begin{tabular}{rllllllrll}
\hline & \multicolumn{2}{c}{$\ell^{-}=0=\ell^{+}$} & \multicolumn{2}{c}{$\ell^{-}=0.5=\ell^{+}$} & \multicolumn{2}{c}{$\ell^{-}=1, \ell^{+}=0.5$} & \multicolumn{2}{c}{$\ell^{-}=0.5, \ell^{+}=1$} \\
\cline { 2 - 9 }$n$ & $k_{n}$ & $A_{n}$ & $k_{n}$ & $A_{n}$ & $k_{n}$ & \multicolumn{1}{c}{$A_{n}$} & \multicolumn{1}{l}{$k_{n}$} & $A_{n}$ \\
\hline 1 & 1.5708 & 1.0320 & 1.0769 & 1.7895 & 0.9631 & 1.7878 & 0.9631 & 2.2363 \\
2 & 3.1416 & 0.0000 & 2.2889 & 0.0000 & 2.1609 & -0.0179 & 2.1609 & 0.0290 \\
3 & 4.7124 & 0.0382 & 3.6436 & 0.0172 & 3.5367 & 0.0086 & 3.5367 & 0.0155 \\
4 & 6.2832 & 0.0000 & 5.0870 & 0.0000 & 5.0013 & -0.0005 & 5.0013 & 0.0010 \\
5 & 7.8540 & 0.0083 & 6.5783 & 0.0011 & 6.5085 & 0.0005 & 6.5085 & 0.0009 \\
\hline
\end{tabular}

When $\ell^{-}=\ell^{+}=0$ (that is, no slip on the channel walls), equation (2.7) reduces to $\sin \left(2 k_{n}\right)=0$, which implies that $k_{n}=n \pi / 2$, and equation (2.9) reduces to $A_{n}=$ $16\left[1-(-1)^{n}\right] / n^{3} \pi^{3}$, so that equation (2.8) becomes

$$
u(\hat{y}, t)=\sum_{n=1}^{\infty} \frac{16}{n^{3} \pi^{3}}\left[1-(-1)^{n}\right] \sin \left(\frac{n \pi \hat{y}}{2}\right) e^{-n^{2} \pi^{2} t / 4},
$$

which corresponds to the classical solution. When $\ell^{-}=\ell^{+}=\ell$ (equal slip on the channel walls), equation (2.7) reduces to

$$
\tan \left(2 k_{n}\right)-\frac{2 k_{n} \ell}{\ell^{2} k_{n}^{2}-1}=0,
$$

and the left-hand side of (2.9) reduces to $A_{n}\left(k_{n}^{2} \ell^{2}+\ell+1\right)$, as in the book by Hill and Dewynne [8, Ch. 4].

2.3. Complete solution The complete velocity profile is given by

$$
\begin{aligned}
v_{x}(y, t)= & \frac{3\left(\ell^{-}+\ell^{+}\right)+4 \ell^{-} \ell^{+}+2}{\ell^{-}+\ell^{+}+2}-\frac{2\left(\ell^{-}-\ell^{+}\right)}{\ell^{-}+\ell^{+}+2} y-y^{2} \\
& -\sum_{n=1}^{\infty} A_{n}\left\{\sin \left[k_{n}(y+1)\right]+\ell^{-} k_{n} \cos \left[k_{n}(y+1)\right]\right\} e^{-k_{n}^{2} t},
\end{aligned}
$$

where the eigenvalues $k_{n}$ are obtained from equation (2.7) and arranged such that $0<k_{1}<k_{2}<\cdots$ and the constants $A_{n}$ are determined from equation (2.9). Table 1 shows the first five values of $k_{n}$ (obtained by bisection) and $A_{n}$ for various amounts of slip. Notice that for equal slip lengths the coefficients $A_{n}$ are nonzero for odd values of $n$ (as for no slip), and that for nonzero slip lengths the coefficients $A_{n}$ approach zero more rapidly than in the no-slip case, indicating that the Fourier series solution converges more rapidly with the presence of slip.

\section{Numerical modelling}

Consider the unsteady flow of an incompressible Newtonian fluid between two parallel planes of length 0.1 units (so $L=0.1$ ) separated by a distance of 0.02 units 
(so $h=0.01$ ). Initially the fluid is at rest, and is set in motion by a suddenly imposed and maintained pressure difference $P_{0}-P_{L}$ acting over the entire length parallel to the planes, where without loss of generality we set $P_{L}=0$. A Cartesian coordinate system $(x, y)$ is used, where $y$ is measured from the midpoint between the two planes, normal to the planes, while $x$ is measured parallel to the planes and is positive in the direction of flow.

The flow is given by solving the unsteady Navier-Stokes and continuity equations

$$
\rho \frac{\partial \mathbf{v}}{\partial t}+\rho \mathbf{v} \cdot \nabla \mathbf{v}=-\nabla P+\mu \nabla^{2} \mathbf{v}, \quad \nabla \cdot \mathbf{v}=0
$$

where $\mathbf{v}=\left(v_{x}, v_{y}\right)$ is the velocity vector, $P$ is pressure, and $\rho$ and $\mu$ are the fluid density and viscosity, respectively. At the channel inlet and outlet, $x=0$ and $x=0.1$, we specify the pressure and require zero vertical velocity component; that is, the boundary conditions are

$$
\begin{aligned}
& P(0, y, t)=P_{0}, \quad v_{y}(0, y, t)=0, \\
& P(0.1, y, t)=0, \quad v_{y}(0.1, y, t)=0 .
\end{aligned}
$$

On the channel walls we apply Navier slip conditions

$$
v_{n}=0, \quad v_{t}=-\frac{\ell}{\mu} T_{t},
$$

where $v_{n}$ and $v_{t}$ are the normal and tangential velocity components, $T_{t}$ is the tangential stress, and $\ell$ is the slip length. Taking $\ell=0$ yields the no-slip condition, while the case of infinite slip lengths corresponds to a potential flow situation. Note that $T_{t}=\mathbf{T} \cdot \mathbf{t}$, where $\mathbf{T}=\boldsymbol{\sigma} \cdot \mathbf{n}$ is the stress vector for the unit outward normal $\mathbf{n}$ to the surface and $\boldsymbol{\sigma}=-P \mathbf{I}+\mu\left[\boldsymbol{\nabla} \mathbf{v}+(\boldsymbol{\nabla} \mathbf{v})^{\top}\right]$ is the stress tensor.

To solve for the flow numerically and allow direct comparison with the analytical solution (2.10), we first nondimensionalize the governing equations and boundary conditions so they match with the previous analytical solution; that is, we use the same nondimensionalization as in the previous section. By setting the density $\rho$ to a convenient value (in this case $1000 \mathrm{~kg} / \mathrm{m}^{3}$, the density of water), from the time scale in (2.2) we set $\mu / \rho h^{2}=1$ so that $\mu=h^{2} \rho$. From the velocity scale we set $A h^{2} / 2 \mu=1$ so that $A=2 \rho$, which implies from (2.1) and (3.1) that $P_{0}=2 L \rho$. Finally, from the slip length scale and (3.2) we scale the slip lengths here with $\rho h$. Hence, on the channel walls we have the Navier slip condition

$$
v_{t}=-\frac{\ell}{\rho h} T_{t},
$$

where the value of the slip length $\ell$ may be different for each wall.

We solve the dimensionless problem using COMSOL Multiphysics (Version 3.5a) [4], hereafter referred to as COMSOL, and a mixed finite element formulation with first- and second-order Lagrange elements for the pressure and velocity, respectively. 
3.1. Implementing the Navier slip conditions The slip boundary conditions on the channel walls are implemented via a penalty approach that is described and verified by Stokes and Carey [17] for steady Stokes and Navier-Stokes flow but has yet to be verified for unsteady flows. The penalty method places no limitations on boundary shape, handles no-slip and no-stress boundaries as limiting cases of Navier slip and partial penetration, and is relatively straightforward to formulate and implement in a finite element code, such as COMSOL [4].

Consider the boundary conditions

$$
\epsilon_{n} T_{n}+v_{n}=0, \quad \epsilon_{t} T_{t}+v_{t}=0,
$$

where $T_{t}$ and $T_{n}$ denote the tangential and normal components of stress, respectively, $v_{t}$ and $v_{n}$ denote the tangential and normal velocity components, and $\epsilon_{t}$ and $\epsilon_{n}$ are positive constants. As shown by Stokes and Carey [17], these are the natural boundary conditions associated with the mixed form Lagrangian for the steady Stokes equations in two spatial dimensions, given by

$$
\begin{aligned}
L_{\epsilon}(\mathbf{v}, p)= & \int_{\Pi}\left[\frac{\mu}{2} \frac{\partial v_{i}}{\partial x_{j}}\left(\frac{\partial v_{i}}{\partial x_{j}}+\frac{\partial v_{j}}{\partial x_{i}}\right)-f_{i} v_{i}-p \frac{\partial v_{j}}{\partial x_{j}}\right] d V \\
& +\frac{1}{2 \epsilon_{t}} \oint_{\Gamma} v_{t}^{2} d s+\frac{1}{2 \epsilon_{n}} \oint_{\Gamma} v_{n}^{2} d s
\end{aligned}
$$

where repeated indices imply summation, $\left(v_{x}, v_{y}\right)=\left(v_{1}, v_{2}\right), \Pi$ denotes the computational domain, which has boundary $\Gamma$, and $\epsilon_{t}$ and $\epsilon_{n}$ are (positive) penalty parameters. The two boundary integral penalty terms are additional to the usual Lagrangian. By setting both $\epsilon_{t}$ and $\epsilon_{n}$ to sufficiently small values, the boundary conditions (3.3) are equivalent to the no-slip conditions $v_{n}=0$ and $v_{t}=0$, now imposed via a penalty formulation of the viscous flow problem. Keeping $\epsilon_{n}$ small and setting $\epsilon_{t}=\ell / \rho h$, we have the no-penetration $\left(v_{n}=0\right)$ and Navier slip conditions. It should be noted that the natural boundary condition associated with the mixed form Lagrangian for the unsteady Navier-Stokes equations in two spatial dimensions is identical to that for the steady Stokes equations in two spatial dimensions.

The boundary conditions (3.3) may be written in terms of Cartesian components to yield

$$
\begin{aligned}
& T_{x}=-\frac{v_{n}}{\epsilon_{n}} n_{x}-\frac{v_{t}}{\epsilon_{t}} t_{x}=-\frac{\left(v_{x} n_{x}+v_{y} n_{y}\right) n_{x}}{\epsilon_{n}}-\frac{\left(v_{x} t_{x}+v_{y} t_{y}\right) t_{x}}{\epsilon_{t}}, \\
& T_{y}=-\frac{v_{n}}{\epsilon_{n}} n_{y}-\frac{v_{t}}{\epsilon_{t}} t_{y}=-\frac{\left(v_{x} n_{x}+v_{y} n_{y}\right) n_{y}}{\epsilon_{n}}-\frac{\left(v_{x} t_{x}+v_{y} t_{y}\right) t_{y}}{\epsilon_{t}} .
\end{aligned}
$$

Conditions (3.4) and (3.5) are implemented in COMSOL via general stress boundary conditions on each of the channel walls.

3.2. Verification of the numerical method To verify the numerical method, we consider the velocity at the centre of the computational domain (at $x=0.05, y=0$ ). 
TABLE 2. Velocity at the centre of the channel, showing convergence with increasing number of mesh elements for $\rho=1000 \mathrm{~kg} / \mathrm{m}^{3}, t=10$ and all penalty parameters set to $10^{-9}$. The mesh element distribution is nonuniform, with a higher density of elements around the boundary and at the midpoint. The velocity from the analytical solution is unity.

\begin{tabular}{ll}
\hline Number of elements & $\bar{v}_{x}$ \\
\hline 124 & 1.000006 \\
496 & 1.000005 \\
1984 & 1.000006 \\
7936 & 1.000005 \\
\hline
\end{tabular}

With a no-slip condition at the channel walls and for large times we should find that the $x$ component of the velocity at the centreline is unity, consistent with the classic steady-state velocity profile $1-y^{2}$. We should also find that the $y$ component of the velocity is zero throughout the computational domain, which is found to be the case for all computations.

Setting $\rho=1000 \mathrm{~kg} / \mathrm{m}^{3}$ and all penalty parameters to $10^{-9}$ (that is, no slip and no penetration on the channel walls), we first checked for convergence by increasing the number of mesh elements for $t=10$ (assumed to correspond to steady state). A nonuniform distribution of mesh elements was used, with a higher density of elements at the boundaries and around the midpoint of the computational domain (the main area where we compare the analytical and numerical solutions). An example of the mesh used with 496 mesh elements is shown in Figure 1. Table 2 shows the velocity at the centre, $\bar{v}_{x}$, obtained with different numbers of mesh elements. It can be seen that convergence is achieved almost immediately with five-figure accuracy for all numbers of elements tested. Given these results, we conclude that simulations using meshes of around 2000 elements will give good accuracy of at least five significant figures and provide reasonable computational time for the unsteady simulations to follow.

Next we wish to establish the magnitude of the penalty parameters $\epsilon_{t}$ and $\epsilon_{n}$ equivalent to no-slip/penetration boundary conditions. In theory, the smaller $\epsilon_{t}\left(\epsilon_{n}\right)$ the closer the boundary condition will be to no slip (no penetration), but as discussed by Matthews and Stokes [13], too small a value will result in error due to loss of precision. Again setting $\rho=1000 \mathrm{~kg} / \mathrm{m}^{3}$, and with a mesh of 1984 elements, we obtained values for $\bar{v}_{x}$ with $\epsilon_{t}=\epsilon_{n}=\epsilon$ and $10^{-9} \leq \epsilon \leq 10^{-5}$ on each of the channel walls for $t=10$, as shown in Table 3 . The results are, to four decimal places, identical for $\epsilon \leq 10^{-8}$; they are also, to four decimal places, identical to those obtained with no-slip boundary conditions on the channel walls set in the standard way (not shown, but see Section 4). For this investigation we adopted $\epsilon_{t}, \epsilon_{n}=10^{-9}$ as satisfactory for no slip/penetration.

With a Navier slip condition at the channel walls, with equal slip lengths and for large times we should find that the velocity at the centreline is equal to $1+2 \ell$, consistent with the steady-state slip velocity profile $1+2 \ell-y^{2}$ obtained by Matthews 
TABLE 3. Velocity at the centre of the channel for $\epsilon_{t}=\epsilon_{n}=\epsilon, 10^{-9} \leq \epsilon \leq 10^{-5}$, with $\rho=1000 \mathrm{~kg} / \mathrm{m}^{3}$, $t=10$.

\begin{tabular}{ll}
\hline$\epsilon$ & $\bar{v}_{x}$ \\
\hline $10^{-5}$ & 1.056149 \\
$10^{-6}$ & 1.005334 \\
$10^{-7}$ & 1.000530 \\
$10^{-8}$ & 1.000053 \\
$10^{-9}$ & 1.000006 \\
\hline
\end{tabular}

TABLE 4. Velocity at the centre of the channel, showing convergence with increasing number of mesh elements for $\rho=1000 \mathrm{~kg} / \mathrm{m}^{3}, t=10$ and penalty parameters set to $\epsilon_{n}=10^{-9}$ and $\epsilon_{t}=1$ on both channel walls. The velocity from the analytical solution is 3 .

\begin{tabular}{ll}
\hline Number of elements & $\bar{v}_{x}$ \\
\hline 124 & 2.998403 \\
496 & 2.997436 \\
1984 & 2.998956 \\
7936 & 2.997988 \\
\hline
\end{tabular}

and Hill [11]. Setting $\rho=1000 \mathrm{~kg} / \mathrm{m}^{3}, \epsilon_{n}=10^{-9}$ (that is, no penetration on the channel walls) and $\epsilon_{t}=1 / \rho h$ (slip on the channel walls with unity slip lengths), we again checked for convergence by increasing the number of mesh elements for $t=10$ (assumed to correspond to steady state). Table 4 shows the velocity at the centre, $\bar{v}_{x}$, obtained with different numbers of mesh elements. It can be seen that again convergence is achieved almost immediately with four-figure accuracy for all numbers of elements tested.

Finally, we establish the magnitude of the penalty parameter $\epsilon_{n}$ equivalent to no penetration boundary conditions. Again setting $\rho=1000 \mathrm{~kg} / \mathrm{m}^{3}$ and $\epsilon_{t}=1 / \rho h$, and with a mesh of 1984 elements, we obtained values for $\bar{v}_{x}$ with $10^{-9} \leq \epsilon_{n} \leq 10^{-5}$ on the channel walls for $t=10$, as shown in Table 5. Again we find that $\epsilon_{n}=10^{-9}$ is satisfactory for no penetration with partial slip.

This completes our verification of our numerical method, and the penalty implementation of the boundary conditions in particular. We now proceed to look at the effect of slip on the flow and compare the analytical solution with the timedependent numerical simulations.

\section{Results and discussion}

For the analytical solution given by equation (2.10), it was found that 25 terms were enough to ensure five-digit accuracy at all times and slip lengths considered. The 
TABLE 5. Velocity at the centre of the channel for $\epsilon_{t}=1$ and $10^{-9} \leq \epsilon \leq 10^{-5}$, with $\rho=1000 \mathrm{~kg} / \mathrm{m}^{3}, t=10$.

\begin{tabular}{ll}
\hline$\epsilon$ & $\bar{v}_{x}$ \\
\hline $10^{-5}$ & 3.236910 \\
$10^{-6}$ & 3.020071 \\
$10^{-7}$ & 3.000908 \\
$10^{-8}$ & 2.999134 \\
$10^{-9}$ & 2.998956 \\
\hline
\end{tabular}

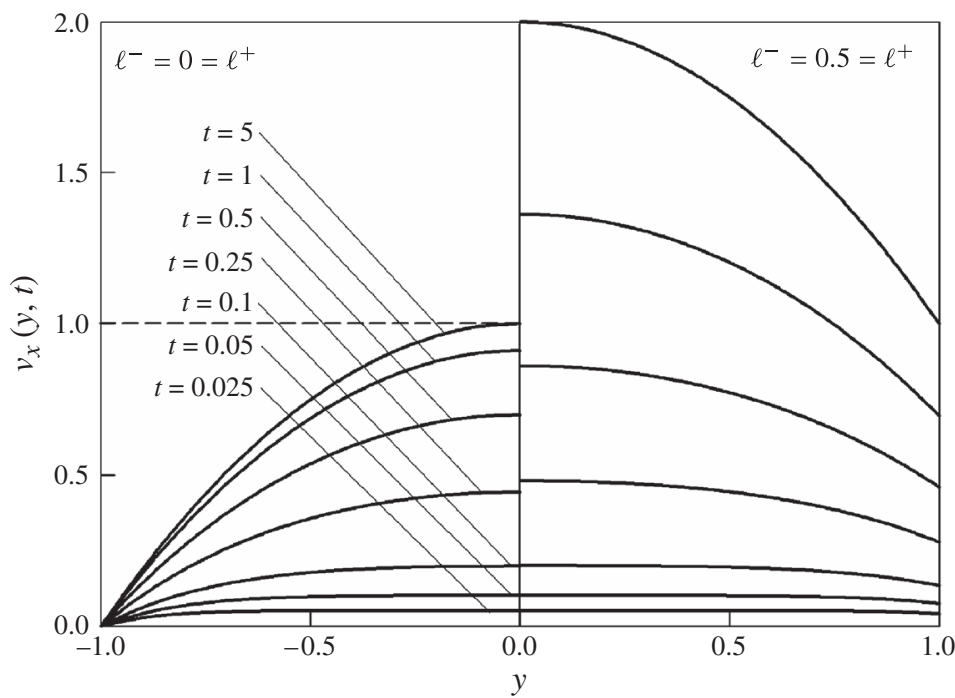

FIGURE 2. Velocity profiles for the analytical and numerical solutions for various times, for no slip $\left(\ell^{-}=0=\ell^{+}\right)$and symmetric slip with $\ell^{-}=0.5=\ell^{+}$. The times for the slip solutions are the same as for the no-slip solutions. The analytical and numerical solutions are indistinguishable at the scale of the plot.

velocity profiles obtained from the analytical solution are shown in Figures 2 and 3 for various times and amounts of slip.

Figure 2 shows the velocity profiles from the analytical solution for times ranging from $t=0.025$ to $t=5$ for no slip (that is, $\ell^{-}=0=\ell^{+}$) and for symmetric slip with $\ell^{-}=0.5=\ell^{+}$. The profiles are symmetric about the centreline $y=0$, so only half the profile is shown for each case. The slip profiles are for the same times as the no-slip profiles. It can be seen that the velocity profile approaches the fully developed solution as time increases and, at the graphical scale used, is essentially the same as the fully developed solution ( $1-y^{2}$ for no slip, $2-y^{2}$ for slip; see (2.6)) by $t=5$.

Figure 3 shows the velocity profiles from the analytical solution for times ranging from $t=0.025$ to $t=5$ for the case of nonsymmetric slip corresponding to $\ell^{-}=1$ 


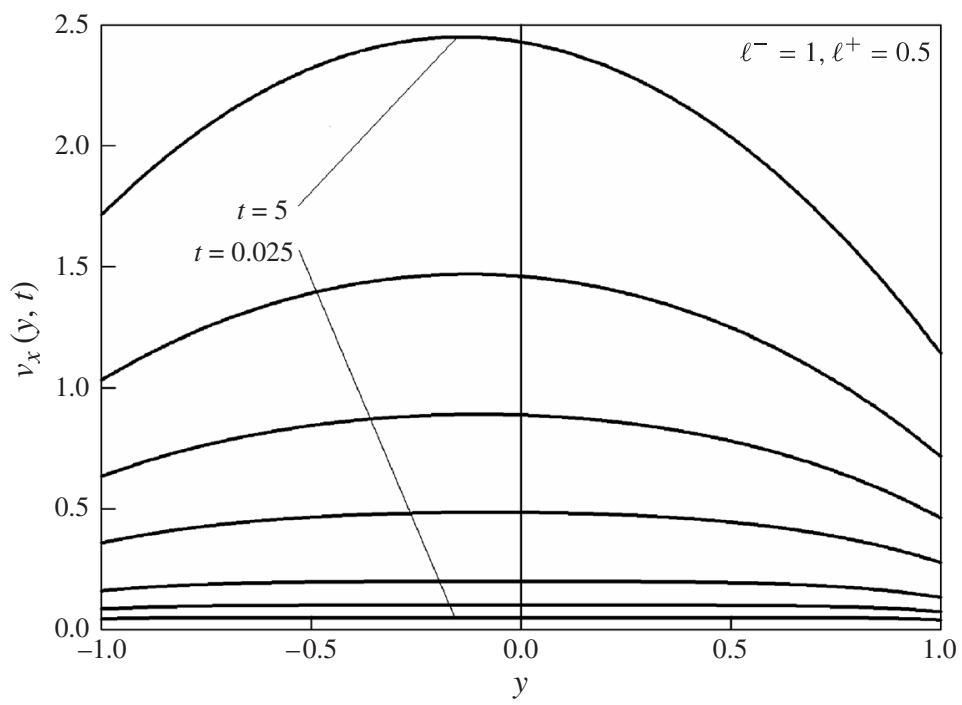

FIGURE 3. Velocity profiles for the analytical and numerical solutions for various times and nonsymmetric $\operatorname{slip}\left(\ell^{-}=1\right.$ and $\left.\ell^{+}=0.5\right)$. The times for the slip solutions are from $t=0.025$ to $t=5$, and are the same as those for the no-slip solutions shown on the left-hand side of Figure 2. The analytical and numerical solutions are indistinguishable at the scale of the plot.

and $\ell^{+}=0.5$. Notice that the profiles are no longer symmetric about the centreline $y=0$. The profiles are for the same times as for the no-slip profiles, shown on the left-hand side of Figure 2. It can be seen again that the profiles gradually increase in magnitude, and that for $t=5$ the profiles are essentially the same as that for the fully developed flow, which from equation (2.6) corresponds to $17 / 7-2 y / 7-y^{2}$ with a maximum at $y=-1 / 7$. Note that the case $\ell^{-}=0.5, \ell^{+}=1$ is a mirror image of Figure 3 about the centreline $y=0$.

The velocity profiles from the numerical modelling were calculated along various perpendicular cross sections of the channel, and they were found to be identical. For the same slip length combinations used in the analytical studies, the velocity profiles were found to be indistinguishable at the scale of the plots in Figures 2 and 3. To ascertain the precise agreement between the analytical and numerical solutions, the velocities $\bar{v}_{x}$ for various times $t$ ranging from $t=0.025$ to $t=5$ at the midpoint of the channel were calculated from the analytical and numerical solutions, and the results are shown in Tables 6 and 7.

Initially, the case of no slip was investigated. From the analytical solution (2.10), the velocities $\bar{v}_{x}$ (corresponding to $y=0$ ) were calculated for various times $t$ ranging from $t=0.025$ to $t=5$. The velocities were also calculated numerically in COMSOL via standard methods (that is, by enforcing $v_{t}=0$ exactly) and via the penalty method with all penalty parameters set to $10^{-9}$. The results from the three cases are shown in Table 6. It can be seen that there is virtually complete agreement between the 
TABLE 6. Comparison of the velocity at the centre of the channel for various $t$ with the analytical solution and the numerical solutions with $\rho=1000 \mathrm{~kg} / \mathrm{m}^{3}$ found via standard no-slip methods and the penalty method with $\epsilon_{t}=10^{-9}=\epsilon_{n}$.

\begin{tabular}{llll}
\hline$t$ & $\bar{v}_{x}$ (analytical) & $\bar{v}_{x}$ (standard) & $\bar{v}_{x}$ (penalty) \\
\hline 0.025 & 0.0500 & 0.0520 & 0.0520 \\
0.05 & 0.1000 & 0.1019 & 0.1019 \\
0.1 & 0.1977 & 0.1993 & 0.1993 \\
0.25 & 0.4432 & 0.4451 & 0.4450 \\
0.5 & 0.6995 & 0.6998 & 0.6998 \\
1 & 0.9125 & 0.9102 & 0.9102 \\
5 & 1.0000 & 1.0000 & 1.0000 \\
\hline
\end{tabular}

TABLE 7. Comparison of the velocity at the centre of the channel for various $t$ with the analytical solution and the numerical solution with $\rho=1000 \mathrm{~kg} / \mathrm{m}^{3}$ for the cases of symmetric slip and nonsymmetric slip.

\begin{tabular}{lllll}
\hline & \multicolumn{2}{c}{$\ell^{-}=0.5=\ell^{+}$} & \multicolumn{2}{c}{$\ell^{-}=1, \ell^{+}=0.5$} \\
\cline { 2 - 5 }$t$ & $\bar{v}_{x}$ (analytical) & $\bar{v}_{x}$ (penalty) & $\bar{v}_{x}$ (analytical) & $\bar{v}_{x}$ (penalty) \\
\hline 0.025 & 0.0500 & 0.0520 & 0.0500 & 0.0520 \\
0.05 & 0.1000 & 0.1020 & 0.1000 & 0.1020 \\
0.1 & 0.1995 & 0.2013 & 0.1996 & 0.2014 \\
0.25 & 0.4804 & 0.4818 & 0.4843 & 0.4858 \\
0.5 & 0.8619 & 0.8653 & 0.8867 & 0.8895 \\
1 & 1.3626 & 1.3715 & 1.4592 & 1.4663 \\
5 & 1.9938 & 1.9931 & 2.4049 & 2.4052 \\
\hline
\end{tabular}

numerical solutions via the penalty method and the standard method, and agreement to two decimal places with the analytical solution. Although small, the discrepancy between the analytical and numerical solutions is likely due to the entrance and end effects of the channel in the numerical solution, which the analytical solution cannot possibly account for. Indeed, by increasing the channel length $L$ (and adjusting the pressure $P_{0}$ accordingly), it was found that the agreement between the analytical and numerical solution improves as $L$ increases.

Finally, we compare the average velocities $\bar{v}_{x}$ from the analytical and the numerical solutions for $\ell^{-}=0.5=\ell^{+}$and $\ell^{-}=1, \ell^{+}=0.5$ for various times ranging from $t=$ 0.025 to $t=5$. These are shown in Table 7. Again we see good agreement, with the two results the same to two decimal places.

We may conclude from this analysis that the penalty method described and verified by Stokes and Carey [17] for steady Navier-Stokes flow has now been directly verified for unsteady Navier-Stokes flow via direct comparison with an analytical slip solution. 
However, as pointed out by Stokes and Carey [17], implementation of the Navier slip boundary condition is relatively straightforward for wall boundaries that coincide with Cartesian coordinate planes $[9,16]$, which is the case considered here. A complete and thorough verification therefore requires the analysis of a problem involving unsteady flow with an inclined or a curved boundary, along with direct comparison with an analytical solution.

\section{Appendix A. Details of the boundary value problem}

Consider the boundary value problem

$$
\begin{aligned}
& Y^{\prime \prime}(\hat{y})+\lambda Y(\hat{y})=0, \\
& Y(0)-\ell^{-} Y^{\prime}(0)=0, \quad Y(2)+\ell^{+} Y^{\prime}(2)=0 .
\end{aligned}
$$

If $\lambda=-k^{2}<0$ then $Y(\hat{y})=C_{1} e^{k \hat{y}}+C_{2} e^{-k \hat{y}}$, and the boundary conditions imply that for a nontrivial solution to exist we must have

$$
e^{4 k}=\frac{\left(1-\ell^{-} k\right)\left(1-\ell^{+} k\right)}{\left(1+\ell^{-} k\right)\left(1+\ell^{+} k\right)} .
$$

With $\ell^{-}, \ell^{+}>0$ and $k \neq 0$ this equation has no solution, and hence there are no negative eigenvalues. If $\lambda=0$ then $Y(\hat{y})=C_{1} \hat{y}+C_{2}$, and the boundary conditions imply that for a nontrivial solution to exist we must have $\ell^{-}+\ell^{+}+2=0$, which cannot be satisfied for positive slip lengths. Hence zero is not an eigenvalue, and therefore all the eigenvalues are positive.

The fact that all the eigenvalues are positive can also be deduced from the Rayleigh quotient, which for this problem is given by

$$
\lambda=\frac{\int_{0}^{2}\left(Y^{\prime}\right)^{2} d \hat{y}-\left[Y Y^{\prime}\right]_{0}^{2}}{\int_{0}^{2} Y^{2} d \hat{y}}
$$

From the boundary conditions, this may be written as

$$
\lambda=\frac{\int_{0}^{2}\left(Y^{\prime}\right)^{2} d \hat{y}+\ell^{-}\left[Y^{\prime}(0)\right]^{2}+\ell^{+}\left[Y^{\prime}(2)\right]^{2}}{\int_{0}^{2} Y^{2} d \hat{y}},
$$

and it follows that $\lambda>0$.

\section{References}

[1] G. K. Batchelor, An introduction to fluid dynamics (Cambridge University Press, Cambridge, 1967).

[2] B. Bhushan, J. N. Israelachvili and U. Landman, "Nanotribology: friction, wear and lubrication at the atomic scale", Nature 374 (1994) 607-616; doi:10.1038/374607a0.

[3] H. S. Carslaw and J. C. Jaeger, Conduction of heat in solids, 2nd edn (Oxford University Press, New York, 1986). 
[4] "COMSOL AB, COMSOL Multiphysics (Version 3.5a)", 2008; http://www.comsol.com.

[5] M. Gad-el-Hak, "The fluid mechanics of microdevices-The Freeman scholar lecture", J. Fluids Engng 121 (1999) 5-33; doi:10.1115/1.2822013.

[6] M. Gad-el-Hak, "Transport phenomena in microdevices", Z. Angew. Math. Mech. 84 (2004) 494498; doi:10.1002/zamm.200310118.

[7] S. Granick, "Motions and relaxations of confined liquids", Science 253 (1991) 1374-1379; doi:10.1126/science.253.5026.1374.

[8] J. M. Hill and J. N. Dewynne, Heat conduction (Blackwell Scientific, London, 1987).

[9] V. John, "Slip with friction and penetration with resistance boundary conditions for the NavierStokes equations-numerical tests and aspects of the implementation", J. Comput. Appl. Math. 147 (2002) 287-300; doi:10.1016/S0377-0427(02)00437-5.

[10] G. Karniadakis, A. Beskok and N. Aluru, Microflows and nanoflows: fundamentals and simulation (Springer, New York, 2005).

[11] M. T. Matthews and J. M. Hill, "Newtonian flow with nonlinear Navier boundary condition", Acta Mechanica 191 (2007) 195-217; doi:10.1007/s00707-007-0454-8.

[12] M. T. Matthews and J. M. Hill, "Effect of slip on the linear stability of flow through a tube", Z. Angew. Math. Phys. 59 (2008) 360-379; doi:10.1007/s00033-007-7116-5.

[13] M. T. Matthews and Y. M. Stokes, "Lubrication analysis and numerical simulation of the viscous micropump with slip", Int. J. Heat Fluid Flow 33 (2012) 22-34; doi:10.1016/j.ijheatfluidflow.2011.09.009.

[14] J. C. Maxwell, "On stresses in rarified gases arising from inequalities of temperature", Phil. Trans. R. Soc. Lond. 170 (1879) 231-256; doi:10.1098/rstl.1879.0067.

[15] C. L. M. H. Navier, "Mémoire sur les lois du mouvement des fluides", Mémoires de la Classe des Sciences Mathématiques et Physiques de l'Institut de France 6 (1823) 389-440.

[16] T. Qian, X.-P. Wang and P. Sheng, "A variational approach to moving contact line hydrodynamics", J. Fluid Mech. 564 (2006) 333-360; doi:10.1017/S0022112006001935.

[17] Y. M. Stokes and G. F. Carey, "On generalised penalty approaches for slip, free surface and related boundary conditions in viscous flow simulation", Internat. J. Numer. Methods Heat Fluid Flow 21 (2011) 668-702; doi:10.1108/09615531111148455.

[18] C. Y. Wang, "Exact solutions of the unsteady Navier-Stokes equations", Appl. Mech. Rev. 42 (1989) S269-S282; doi:10.1115/1.3152400.

[19] C. Y. Wang, "Two-fluid oscillatory flow in a channel", Theor. Appl. Mech. Lett. 1 (2011) 032007; doi:10.1063/2.1103207. 